\title{
On The SLOpe STRATification OF CERTAin SHImura VARIETIES
}

\author{
CHIA-Fu YU ${ }^{1}$
}

\begin{abstract}
In this paper we study the slope stratification on the good reduction of the type $\mathrm{C}$ family Shimura varieties. We show that there is an open dense subset $U$ of the moduli space such that any point in $U$ can be deformed to a point with a given lower admissible Newton polygon. For the Siegel moduli spaces, this is obtained by F. Oort which plays an important role in his proof of the strong Grothendieck conjecture concerning the slope stratification. We also investigate the $p$-divisible groups and their isogeny classes arising from the abelian varieties in question.
\end{abstract}

\section{$\S 1$. Introduction}

Let $p$ be a rational prime number. Let $B$ be a finite-dimensional division algebra over $\mathbb{Q}$ with a positive involution $*$. We consider the cases where $B$ is either

(a) a totally real field $F$ of degree $d$, or

(b) a totally indefinite quaternion algebra over a totally real field $F$ of degree $d$.

Let $O_{B}$ be an order of $B$ stable under the involution such that $O_{B} \otimes \mathbb{Z}_{p}$ is a maximal order of $B \otimes \mathbb{Q}_{p}$. Let $\mathcal{M}$ denote the moduli stack over $\overline{\mathbb{F}}_{p}$ of separably polarized abelian $O_{B}$-varieties of dimension $g$, where $g=d n$ in case (a) and $g=2 d n$ in case (b) for some positive integer $n$. A polarized abelian $O_{B}$-variety $(A, \lambda, \iota)$ is a polarized abelian variety $(A, \lambda)$ together with a ring embedding $\iota: O_{B} \rightarrow \operatorname{End}(A)$ such that $\lambda \iota\left(b^{*}\right)=\iota(b)^{t} \lambda$ for all $b \in O_{B}$. For a polarized abelian $O_{B}$-variety $\underline{A}=(A, \lambda, \iota)$, the associated $p$-divisible group $\underline{H}=\underline{A}\left[p^{\infty}\right]$ with the additional structure is a quasi-polarized $p$-divisible $O_{B} \otimes \mathbb{Z}_{p}$-group (in [26]), or a quasi-polarized BT $O_{B} \otimes \mathbb{Z}_{p}$-module (in [12]). Define the (refined) Newton polygon of $\underline{H}$ by

$$
N P(\underline{H})=\left(N P\left(H_{v}\right)\right)_{v \mid p},
$$

where $H=\oplus_{v} H_{v}$ is the decomposition of $p$-divisible groups coming from $O_{F} \otimes \mathbb{Z}_{p}=\oplus_{v} O_{v}$ and $N P\left(H_{v}\right)$ is the usual Newton polygon of $H_{v}$. A natural question is whether a given quasi-polarized $p$-divisible $O_{B} \otimes \mathbb{Z}_{p^{-}}$group over an algebraically closed field $k$ comes from a quasi-polarized abelian $O_{B}$-variety. Let $M$ be the covariant Dieudonné module associated to $\underline{H}$ and let $M=\oplus_{v \mid p} M_{v}$ the corresponding decomposition. One can show that each factor $M_{v}$, considered as an $O_{v} \otimes W(k)$ module, is free. If $M$ arises from an abelian $O_{B}$-variety, then it satisfies an additional condition that the ranks $\operatorname{rank}_{O_{v} \otimes W(k)} M_{v}$ are the same for all $v \mid p$. The latter is equivalent to the following condition:

$(*) \quad \operatorname{Tr}_{W(k)}(a ; M) \cdot[F: \mathbb{Q}]=\left(\operatorname{rank}_{W(k)} M\right) \cdot \operatorname{Tr}_{F / \mathbb{Q}}(a), \quad$ for all $a \in O_{F}$

In this paper we prove

(1.1) Theorem Let $k$ be an algebraically closed field of characteristic $p$.

(1) Any quasi-polarized supersingular p-divisible $O_{B} \otimes \mathbb{Z}_{p}$-group over $k$ whose Dieudonné module satisfies $(*)$ is realized by a quasi-polarized abelian $O_{B}$-variety $\underline{A}$.

\footnotetext{
${ }^{1}$ November 23, 2018
} 
(2) Let $\underline{H}$ be a quasi-polarized p-divisible $O_{B} \otimes \mathbb{Z}_{p}$-group over $k$. If its Newton polygon $N P(\underline{H})$ is realized by an object $\underline{A}^{\prime}$, then $\underline{H}$ is realized by an object $\underline{A}$.

The statement (2) is an extension of (a special case of) a result of Rapoport and Richartz [22, Proposition 1.17] on the Newton map. The statement (1) follows from (2) and a construction of supersingular points; see Theorem 2.5 for the statement. The existence of supersingular points is well-known, at least for some special cases. We simply supply a more complete reference here.

A Newton polygon $\underline{\beta}=\left(\beta_{v}\right)_{v}$ is called admissible if it arises from a quasi-polarized $p$-divisible $O_{B} \otimes \mathbb{Z}_{p}$-group $\underline{H}$ satisfying $(*)$. The partial order imposed on the set of admissible Newton polygons here is given by $\left(\beta_{v}\right)_{v} \geq\left(\beta_{v}^{\prime}\right)_{v}$ if every component $\beta_{v}$ lies above or equal to $\beta_{v}^{\prime}$. In other words, the associated Newton stratum with higher Newton polygon has smaller dimension. For the readers who are familiar with Shimura varieties, the admissible Newton polygons correspond exactly the elements in the image of the set $B(G, \mu)$ (e.g. in [21, Section 4]) of $\mu$-admissible $\sigma$-conjugacy classes under the Newton map, where $G$ is the base change to $\mathbb{Q}_{p}$ of the group defining Shimura varieties and $\mu$ is the corresponding dominant minuscule coweight.

Let $\Delta$ be the product of rational primes at which $B$ is ramified.

(1.2) Theorem Assume that $p \nmid \Delta$. Let $U$ be an open subset of $\mathcal{M}$ defined by

$$
U=\left\{\underline{A}=(A, \lambda, \iota) \in \mathcal{M}\left|a\left(H_{v}\right) \leq 1, \forall v\right| p\right\}
$$

where $H_{v}$ is the $v$-component of $\underline{A}\left[p^{\infty}\right], a\left(H_{v}\right):=\operatorname{dim}_{k} \operatorname{Hom}\left(\alpha_{p}, H_{v}\right)$ is the a-number of $H_{v}$, and $v$ runs through the primes of $O_{F}$ over $p$.

Then for any point $u \in U$ with Newton polygon $\beta$ and for any admissible Newton polygon $\beta^{\prime}$ below $\underline{\beta}$ there is a generalization $u^{\prime} \in \mathcal{M}$ of $u$ whose $\bar{N}$ ewton polygon is equal to $\underline{\beta}^{\prime}$.

The open subset $U$ is dense in $\mathcal{M}$ as it contains the ordinary locus. When $B=\mathbb{Q}$, Theorem 1.2 is proved by F. Oort [16, which plays an important role in his proof of the strong Grothendieck conjecture for the Siegel moduli space [17]: replacing $U$ by the total space $\mathcal{M}$ in Theorem 1.2. See Rapoport [20, Section 5] and [21, Section 7] for further discussions. For the present cases, this result is not enough to conclude the analogue of the strong Grothendieck conjecture, as the intersection of a Newton stratum $\mathcal{W}$ with $U$ is not dense in $\mathcal{W}$ in general 25. Nevertheless, Theorem 1.2 concludes a few new results and improves some previous results in the type $\mathrm{C}$ family of Shimura varieties.

(1.3) Theorem Assume that $p \nmid \Delta$. For any sequence of admissible Newton polygons $\beta_{1}>\beta_{2}>$ $\cdots>\beta_{s}$, there is a chain of irreducible substacks $V_{1} \subset V_{2} \subset \cdots \subset V_{s}$ of $\mathcal{M}$ such that the Newton polygon $N P\left(\underline{A}_{\eta_{i}}\right)$ of the generic point $\eta_{i}$ is $\underline{\beta_{i}}$.

This is a weak form of the analogue of the strong Grothendieck conjecture (or called the weak Grothendieck conjecture in Oort [16]). An immediate consequence of this is that every Newton stratum is non-empty. This proves a special case of Conjecture 7.1 in Rapoport [21].

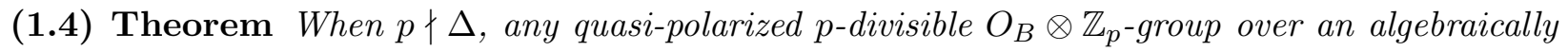
closed field of characteristic p satisfying $(*)$ is realized by a quasi-polarized abelian $O_{B}$-variety. 
When $\mathcal{M}$ is smooth, Theorem 1.4 shows that any $p$-adic invariant stratum arising from $p$ divisible groups in question is non-empty. This improves previous results on stratifications in a special case (type C) by removing the non-emptiness assumption; for example, see the main theorem (Corollary 3.1.6) in Moonen [12. One would expect that Theorem [1.4 holds as well without the condition $p \nmid \Delta$. This is a technical point as the proof presented here replies on an explicit deformation computation, which is more involved in the ramified cases. We hope to return to this technical issue soon.

Finally we remark that the strong Grothendieck conjecture has been proved by Bültel and Wedhorn [1] in the case of inner forms of groups $\mathrm{U}(n, 1)$ associated to imaginary quadratic fields at unramified primes $p \neq 2$, where the split case has been known for a long time as the Lubin-Tate formal moduli.

The paper is organized as follows. In Section 2 we construct a supersingular abelian variety with the additional structure; the basic tool is the classification of positive involutions up to conjugate. In Section 3 we show that the formal isogenies in question are determined by the Newton polygons. In Sections 2 and 3 there is no assumption of $p$. For the rest (Sections 4 and 5) we assume that $p \nmid \Delta$. Using the Morita equivalence, one reduces to considering the deformation problem on local components of $p$-divisible groups in question. We treat the reduced local situation directly. In Section 4 we classify the Dieudonné modules, with the additional structure, with $a$-number one. In Section 5 we recall the Cartier-Dieudonné theory and the method of Cayley-Hamilton. Then we construct the universal deformation and compute any formal completion of any Newton stratum on the open subset $U$ in Theorem 1.2 .

An experienced reader would find that the present formulation differs a bit from the literatures [9, 22, 2, 21]. The choice is simply because it is more direct to adopt the explicit deformation theory of displays and the Cayley-Hamilton method. As the groups defining Shimura varieties treated here satisfies the Hasse principle, the translation is straightforward.

Acknowledgments. The author is deeply indebted to Frans Oort for his inspiring work [16]. An earlier draft (MPI-preprint) of this paper was done during the author's stay at the Max-PlanckInstitut für Mathematik in Bonn in the 2001 Summer. He wishes to thank the Institute for the kind hospitality and the excellent working environment. One should mention that Rapoport's article 21] has been very helpful when the author reorganizes the present paper. Finally, he acknowledges the referee for his encourgements and helpful comments.

\section{$\S 2$. Construction of supersingular points}

In the rest of this paper, $k$ denotes an algebraically closed field of characteristic $p$. We keep the notation of the previous section. In this section we will construct a polarized supersingular abelian $O_{B}$-variety of dimension $g$ over $k$, where $g=d n$ in case (a) and $g=2 d n$ in case (b) for some positive integer $n$.

(2.1) We recall basic results on simple algebras over $\mathbb{Q}$ with involutions (cf. [10]). Let $D$ be a finitedimensional simple algebra with a positive involution $*$. Then we have $\left(D \otimes_{\mathbb{Q}} \overline{\mathbb{Q}}, *\right) \simeq \oplus_{i=1}^{r}\left(L, *_{1}\right)$, where $r=\left[\operatorname{Cent}(D)^{*=1}: \mathbb{Q}\right]$ and $\left(L, *_{1}\right)$ is one of the following three cases:

(A) : $L=M_{n}(\overline{\mathbb{Q}}) \oplus M_{n}(\overline{\mathbb{Q}})$ and $(A, B)^{*_{1}}=\left(B^{t}, A^{t}\right)$, 
(C) : $L=M_{2 n}(\overline{\mathbb{Q}})$ and $*_{1}$ is the standard symplectic involution,

$(\mathrm{BD}): L=M_{n}(\overline{\mathbb{Q}})$ and $*_{1}$ is the transposition.

We also have the corresponding cases for $(D \otimes \mathbb{Q} \mathbb{R}, *):(D \otimes \mathbb{Q} \mathbb{R}, *) \simeq \oplus_{i=1}^{r}\left(N, *_{2}\right)$ for the same $r$, where $\left(N, *_{2}\right)$ is one of the following
$(\mathrm{A}): N=M_{n}(\mathbb{C})$ and $\left(a_{i j}\right)^{* 2}=\left(\bar{a}_{j i}\right)$
(C) $: N=M_{n}(\mathbb{H})$ and $\left(a_{i j}\right)^{*_{2}}=\left(\bar{a}_{j i}\right)$,
$(\mathrm{BD}): N=M_{n}(\mathbb{R})$ and $\left(a_{i j}\right)^{*_{2}}=\left(a_{j i}\right)$.

Here $a \mapsto \bar{a}$ is the standard conjugation.

(2.2) Let ' be another positive involution on $D$. Then there is an positive element $c \in D$ with $c^{*}=c$ such that $x^{\prime}=c x^{*} c^{-1}$ for all $x \in D^{\times}$[10, Lemma 2.11]. Let $U(D, *)_{\mathbb{Q}}$ denote the associated unitary group, which associates to a commutative $\mathbb{Q}$-algebra $R$ the group of $R$-points

$$
U(D, *)(R):=\left\{x \in\left(D \otimes_{\mathbb{Q}} R\right) ; x x^{*}=1\right\} .
$$

Define the algebraic variety $X_{c}$ over $\mathbb{Q}$ by

$$
X_{c}(R):=\left\{x \in\left(D \otimes_{\mathbb{Q}} R\right) ; x x^{*}=c\right\} .
$$

Note that $X_{c}(R) \neq \emptyset$ if and only if $\left(D \otimes R,^{\prime}\right) \simeq(D \otimes R, *)$. One has $X_{c}(\overline{\mathbb{Q}}) \neq \emptyset$ and the group $U(D, *)$ acts simply-transitively on $X_{c}$ from the right. In other words, $X_{c}$ is a right principal homogeneous space under the group $U(D, *)$. Therefore it gives rise to an element $[c]$ in $\mathrm{H}^{1}(\mathbb{Q}, U(D, *))$. Note that the image of $[c]$ in $\mathrm{H}^{1}(\mathbb{R}, U(D, *))$ is trivial.

(2.3) Lemma Notations as above. If $(D, *)$ is of type $(C)$, then $\left(D,^{\prime}\right) \simeq(D, *)$ over $\mathbb{Q}$.

Proof. In this case, the group $U(D, *)$ is semi-simple and simply-connected. It follows from Kneser's theorem and the Hasse principle that $H^{1}(\mathbb{Q}, U(D, *))=0$, hence $\left(D,^{\prime}\right) \simeq(D, *)$ over $\mathbb{Q}$.

(2.4) Let $\left(D_{1}, *_{1}\right)$ and $\left(D_{2}, *_{2}\right)$ be finite-dimensional simple algebras over $\mathbb{Q}$ with positive involutions. Suppose that the fixed fields of the centers are the same, called $F$. For simplicity, assume that $\operatorname{Cent}\left(D_{1}\right) \otimes_{F} \operatorname{Cent}\left(D_{2}\right)$ is a field. We define a new simple algebra with a positive involution by

$$
(D, *):=\left(D_{1} \otimes_{F} D_{2}, *\right),
$$

where $\left(d_{1} \otimes d_{2}\right)^{*}:=d_{1}^{* 1} \otimes d_{2}^{* 2}$. Clearly $*$ is an involution on $D$. To see its positivity, first we have the following formula, for $a \in D_{1}$ and $b \in D_{2}$,

$$
\begin{aligned}
\operatorname{Tr}_{D_{1} \otimes_{F} D_{2} / \mathbb{Q}}(a \otimes b) & =\sum_{\sigma: F \hookrightarrow \overline{\mathbb{Q}}} \operatorname{Tr}_{\left[\left(D_{1} \otimes_{F, \sigma} \overline{\mathbb{Q}}\right) \otimes\left(D_{2} \otimes_{F, \sigma} \overline{\mathbb{Q}}\right)\right] / \overline{\mathbb{Q}}}(\sigma(a) \otimes \sigma(b)) \\
& =\sum_{\sigma: F \hookrightarrow \overline{\mathbb{Q}}} \operatorname{Tr}_{\left(D_{1} \otimes_{F, \sigma} \overline{\mathbb{Q}}\right) / \overline{\mathbb{Q}}}(\sigma(a)) \cdot \operatorname{Tr}_{\left(D_{2} \otimes_{F, \sigma} \overline{\mathbb{Q}}\right) / \overline{\mathbb{Q}}}(\sigma(b))
\end{aligned}
$$


It follows that for $a_{1}, a_{2} \in D_{1}$ and $b_{1}, b_{2} \in D_{2}$,

$$
\begin{aligned}
\operatorname{Tr}_{D_{1} \otimes_{F} D_{2} / \mathbb{Q}}\left(\left(a_{1} \otimes b_{1}\right)\left(a_{2} \otimes b_{2}\right)^{*}\right) & \\
& =\sum_{\sigma: F \hookrightarrow \mathbb{R}} \operatorname{Tr}_{\left(D_{1} \otimes_{F, \sigma} \mathbb{R}\right) / \mathbb{R}}\left(\sigma\left(a_{1}\right) \sigma\left(a_{2}\right)^{*_{1}}\right) \cdot \operatorname{Tr}_{\left(D_{2} \otimes_{F, \sigma} \mathbb{R}\right) / \mathbb{R}}\left(\sigma\left(b_{1}\right) \sigma\left(b_{2}\right)^{*_{2}}\right),
\end{aligned}
$$

which is a positive form. Moreover, we have the following results:

- If one of them is of type $(\mathrm{A})$, then $(D, *)$ is of type $(\mathrm{A})$.

- If both are of type $(\mathrm{C})$ or of type $(\mathrm{BD})$, then $(D, *)$ is of type $(\mathrm{BD})$.

- If one is of type $(\mathrm{C})$ and the other is of type $(\mathrm{BD})$, then $(D, *)$ is of type $(\mathrm{C})$.

(2.5) Theorem There exists a polarized supersingular abelian $O_{B}$-variety of dimension $g$ over $k$.

Proof. Case (a): $B=F,[F: \mathbb{Q}]=d$ and $g=d n$. Choose a supersingular elliptic curve $E$ and put $A_{0}:=\left(E \otimes_{\mathbb{Z}} O_{F}\right)^{n}$. One can choose a polarization on $A_{0}$, induced from one on $E$, and make $A_{0}$ a polarized supersingular abelian $O_{F}$-variety.

Case (b): $[B: F]=4,[F: \mathbb{Q}]=d$ and $g=2 d n$. Choose a supersingular elliptic curve $E$ and put $A_{0}:=\left(E \otimes O_{F}\right)^{2 n}$ as before. Let $\left(D,^{\prime}\right)$ be the simple algebra $\operatorname{End}_{O_{F}}\left(A_{0}\right) \otimes \mathbb{Q}$ with a Rosati involution. Note that $D \simeq M_{2 n}\left(D_{F}\right)$, where $D_{F}:=D_{p} \otimes_{\mathbb{Q}} F$ and $D_{p}$ is the quaternion algebra over $\mathbb{Q}$ ramified exactly at $\{\infty, p\}$. The nontrivial Brauer invariants for $D_{F}$ are $\frac{1}{2}$ at each infinity and $\frac{1}{2}$ at some primes above $p$. Let $C$ be the quaternion algebra over $F$ such that the Brauer invariants of $B \otimes_{F} C$ are the same as those of $D$. Replacing $C$ by $M_{n}(C)$, we have $B \otimes_{F} C \simeq D$. Choose any positive involution on $C$ and make the product positive involution $*$ on $B \otimes_{F} C$, which extends $*$ on $B$. By Lemma 2.3. there is an isomorphism $\left(B \otimes_{F} C, *\right) \simeq\left(D,^{\prime}\right)$. Therefore, we construct a polarized supersingular abelian $O_{F}$-variety $A_{0}$ together with a monomorphism $B \rightarrow \operatorname{End}_{O_{F}}\left(A_{0}\right) \otimes \mathbb{Q}$ preserving the involutions. Finally, one can choose an abelian $O_{B}$-variety in the $B$-linear isogeny class of $A_{0}$ and then take the induced $B$-linear polarization. The construction is complete.

(2.6) Remark There is another way to construct supersingular points as in (2.5). One first constructs a supersingular abelian variety up to isogeny together with a $B$-action. This can be done by choosing an embedding from $B$ to the endomorphism algebra. Then a result of Kottwitz [10. Lemma 9.2] furnishes a $B$-linear polarization. This construction has been used in Bültel and Wedhorn [1, Lemma 5.2].

\section{$\S 3$. Formal isogenies}

We keep the notation as in the previous sections. Theorem 1.1 (2) follows from the following

(3.1) Proposition Let $\underline{A}_{1}, \underline{A}_{2}$ be polarized abelian $O_{B}$-varieties over $k$. If $N P\left(\underline{A}_{1}\right)=N P\left(\underline{A}_{2}\right)$, then there is a polarized $O_{B} \otimes \mathbb{Z}_{p}$-linear quasi-isogeny from $\underline{A}_{1}\left[p^{\infty}\right]$ to $\underline{A}_{2}\left[p^{\infty}\right]$

This means that the quasi-isogeny $\varphi \in \operatorname{Hom}\left(A_{1}\left[p^{\infty}\right], A_{2}\left[p^{\infty}\right]\right) \otimes \mathbb{Q}$ is $O_{B} \otimes \mathbb{Z}_{p}$-linear and that $\varphi^{*} \lambda_{2}=\lambda_{1}$. Since the situation is local, one will replace $F$ by its completion at one prime and $B$ by its completion. Let $H_{1}$ and $H_{2}$ be the corresponding factor of $\underline{A}_{1}\left[p^{\infty}\right]$ and $\underline{A}_{2}\left[p^{\infty}\right]$ respectively. We have two cases, by the Morita equivalence: 
(a) $O_{B}=O_{F}$.

(b) $B$ is a division quaternion algebra over $F$ with an involution $x^{*}=\alpha x^{\prime} \alpha^{-1}$ for some $\alpha \neq 0$ and $\alpha^{\prime}=-\alpha$, where $x \mapsto x^{\prime}$ is the standard involution.

(3.2) Lemma Let $D$ be a finite-dimensional $\mathbb{Q}_{p}$-simple algebra, and $O_{D}$ a maximal order of $D$. Then any two p-divisible $O_{D}$-groups $H_{1}, H_{2}$ with $N P\left(H_{1}\right)=N P\left(H_{2}\right)$ are $O_{D}$-linearly isogenous.

Proof. The proof is elementary; one can use the Noether-Skolem theorem to adjust a chosen isogeny to be $O_{D}$-linear (cf. [25, Section 10]), or use a result of Rapoport and Zink (see Remark 3.4 below) to conclude this. Hence we omit the proof.

Now we choose an $O_{B}$-linear isogeny $\varphi: H_{1} \rightarrow H_{2}$. The pullback of $\lambda_{2}$ has the form $\lambda_{1} a$ for some $a \in \operatorname{End}_{O_{B}}\left(H_{1}\right) \otimes \mathbb{Q}_{p}$ which is invariant under the Rosati involution $*_{1}$ by $\lambda_{1}$. The element $[a]$ defines $J_{1}$-torsor over $\mathbb{Q}_{p}$, where $J_{1}$ is the unitary algebraic group $\operatorname{Aut}_{B}\left(H_{1}, *_{1}\right)$ over $\mathbb{Q}_{p}$. This $J_{1}$-torsor vanishes if and only if there is an element $b \in \operatorname{End}_{O_{B}}\left(H_{1}\right) \otimes \mathbb{Q}_{p}$ such that $b^{*_{1}} b=a$. Note that for $b \in \operatorname{End}_{O_{B}}\left(H_{1}\right)$, the pull-back $b^{*} \lambda_{1}$ is $\lambda_{1} b^{* 1} b$. By Lemma 3.3 below, there is an element $b \in \operatorname{End}_{O_{B}}\left(H_{1}\right) \otimes \mathbb{Q}_{p}$ such that $b^{* 1} b=a^{-1}$. Then one shows $(\varphi b)^{*} \lambda_{2}=\lambda_{1}$. This finishes the proof of Proposition 3.1 .

(3.3) Lemma Let $J_{1}$ be as above. One has $\mathrm{H}^{1}\left(\mathbb{Q}_{p}, J_{1}\right)=0$.

Proof. Let $[H]$ denote the isogeny class of a $p$-divisible group $H$. We write

$$
\left[H_{1}\right]=\oplus_{\lambda<1 / 2}\left[H_{1, \lambda} \oplus H_{1,1-\lambda}\right] \oplus\left[H_{1,1 / 2}\right]
$$

into the slope decomposition. The quasi-polarization sends each factor onto its dual. One has

$$
J_{1}=\prod_{\lambda<1 / 2} J_{1, \lambda} \times J_{1,1 / 2}
$$

where $J_{1, \lambda}$ (resp. $\left.J_{1,1 / 2}\right)$ is the unitary group $\operatorname{Aut}_{B}\left(\left[H_{1, \lambda} \oplus H_{1,1-\lambda}\right], *_{1}\right)\left(\operatorname{resp} . \operatorname{Aut}_{B}\left(\left[H_{1,1 / 2}\right], *_{1}\right)\right)$ over $\mathbb{Q}_{p}$. Therefore, $\mathrm{H}^{1}\left(\mathbb{Q}_{p}, J_{1}\right)=\prod_{\lambda<1 / 2} \mathrm{H}^{1}\left(\mathbb{Q}_{p}, J_{1, \lambda}\right) \times \mathrm{H}^{1}\left(\mathbb{Q}_{p}, J_{1,1 / 2}\right)$.

The group $J_{1, \lambda}$ is the algebraic group associated to the multiplicative group $\operatorname{End}_{B}\left(\left[H_{1, \lambda}\right]\right)^{\times}$. By Hilbert's 90 theorem, $\mathrm{H}^{1}\left(\mathbb{Q}_{p}, J_{1, \lambda}\right)=0$. As the group $J_{1,1 / 2} \otimes \overline{\mathbb{Q}}_{p}$ is isomorphic to a product of symplectic groups, $J_{1,1 / 2}$ is semi-simple and simply connected. This shows $\mathrm{H}^{1}\left(\mathbb{Q}_{p}, J_{1,1 / 2}\right)=0$ and completes the proof.

(3.4) Remark In the proof (3.1) we proved a fact that the fibers of the Newton map are classified by the Galois cohomology $\mathrm{H}^{1}\left(\mathbb{Q}_{p}, J\right)$ for the twisted centralizer $J$. This result has been known due to Rapoport and Richartz [22] for general F-isocrystals with additional structures. Here we simply give an elementary presentation of this result for our special cases. Rapoport and Zink show that $J$ is an inner form of the centralizer of $s \nu(p)$ in the structure group $G_{\mathbb{Q}_{p} s}$, where $\nu$ is the attached slope homomorphism (see [23, 1.7-1.16] for details). From this one can determine $J$ and compute $\mathrm{H}^{1}\left(\mathbb{Q}_{p}, J\right)$ as well. 


\section{$\S 4$. The main theorem}

(4.1) In this and the next sections, $F$ denotes, following the convention, the Frobenius operator on a Dieudonné module. Let $W:=W(k)$ be the ring of Witt vectors, and $\sigma$ the Frobenius map on $W$. Let $\mathcal{O}$ be an étale extension of $\mathbb{Z}_{p}$ of degree $f$, that is, $\mathcal{O} \simeq W\left(\mathbb{F}_{p^{f}}\right)$. A quasi-polarized Dieudonné $\mathcal{O}$-module is a Dieudonné module $M$ together with a non-degenerate alternating $W$-linear pairing (quasi-polarization) $\langle\rangle:, M \times M \rightarrow W$ such that $\langle F x, y\rangle=\langle x, V y\rangle^{\sigma}$ for all $x, y \in M$, and with a ring embedding $\iota: \mathcal{O} \rightarrow \operatorname{End}_{\text {dieu }}(M)$ such that $\langle a x, y\rangle=\langle x, a y\rangle$ for all $a \in \mathcal{O}, x, y \in M$.

Let $I:=\operatorname{Hom}(\mathcal{O}, W)$ be the set of embeddings. We may and do choose an identification $\mathbb{Z} / f \mathbb{Z} \simeq I$, mapping $i \mapsto \sigma_{i}$, so that $\sigma \sigma_{i}=\sigma_{i+1}$.

Let $\underline{M}=(M,\langle\rangle,, \iota)$ be a quasi-polarized Dieudonné $\mathcal{O}$-module over $k$. Denote by

$$
M^{i}:=\left\{x \in M \mid a x=\sigma_{i}(a) x, \forall a \in \mathcal{O}\right\}
$$

the $\sigma_{i}$-component of $M$, which is a free $W$-module of rank $2 r$. We have the decomposition

$$
M=M^{0} \oplus M^{1} \oplus \cdots \oplus M^{f-1}
$$

in which $F: M^{i} \rightarrow M^{i+1}, V: M^{i+1} \rightarrow M^{i}$. The summands $M^{i}, M^{j}$ are orthogonal with respect to the pairing $\langle$,$\rangle for i \neq j$. Conversely, a Dieudonné module together with such a decomposition and these properties is a quasi-polarized Dieudonné $\mathcal{O}$-module.

As $M$ is a free $\mathcal{O} \otimes W$-module, we write $2 g=\operatorname{rank}_{W} M$ and $g=f r$.

(4.2) Lemma Any quasi-polarized Dieudonné $\mathcal{O}$-module $\underline{M}$ has $2 r$ slopes $\lambda_{1} \leq \lambda_{2} \leq \cdots \leq \lambda_{2 r}$ with each slope multiplicity $f$. In other words, the Newton polygon of $M$ satisfies the usual symmetric and integral conditions, and an additional condition that the $x$-coordinate of the breaking points $(x, y)$ is divisible by $f$.

Proof. This should be well-known to experts; we supply a proof for convenience of the reader. Consider the iteration $F^{f}$ of the Frobenius operator on $M$, which preserves each factor $M^{i}$. It follows from Katz's sharp slope estimate $\left[8\right.$ that the lowest slopes of $F^{f}$ on each $M^{i}$ are the same. Taking the exterior powers $\bigwedge^{\bullet} M^{i}$, each $M^{i}$ has the same slope sequence for $F^{f}$. Therefore $M$ has $2 r$ slopes with each slope of multiplicity $f$.

(4.3) Example. When $f=3$ and $r=2$, the possible slope sequences of $M$ have the form $\left\{\lambda_{1}^{3}, \lambda_{2}^{3}, \lambda_{3}^{3}, \lambda_{4}^{3}\right\}$ (with each multiplicity 3 ) and are listed below:

$$
\begin{gathered}
\left(\lambda_{1}, \lambda_{2}, \lambda_{3}, \lambda_{4}\right)=\left(\frac{1}{2}, \frac{1}{2}, \frac{1}{2}, \frac{1}{2}\right)>\left(\frac{1}{3}, \frac{1}{2}, \frac{1}{2}, \frac{2}{3}\right)>\left(\frac{1}{3}, \frac{1}{3}, \frac{2}{3}, \frac{2}{3}\right)> \\
>\left(0, \frac{1}{2}, \frac{1}{2}, 1\right),\left(\frac{1}{6}, \frac{1}{6}, \frac{5}{6}, \frac{5}{6}\right)>\left(0, \frac{1}{3}, \frac{2}{3}, 1\right)>(0,0,1,1) .
\end{gathered}
$$

(4.4) Recall the a-number of a Dieudonné module $M$, denoted by $a(M)$, is the dimension of the $k$-vector space $M /(F, V) M$. We call $M$ local-local if the operators $F$ and $V$ are nilpotent on $M / p M$. For a Dieudonné $\mathcal{O}$-module $\underline{M}$, the a-type of $\underline{M}$ is defined as follows

$$
\underline{a}(\underline{M}):=\left(a_{i}\right)_{i \in I}, \quad a_{i}:=\operatorname{dim}_{k}(M /(F, V) M)^{i} .
$$


(4.5) Theorem Let $\underline{M}$ be a local-local separably quasi-polarized Dieudonné $\mathcal{O}$-module over $k$ such that $\underline{a}(\underline{M})=(1,0, \ldots, 0)$. Then there exists a symplectic basis $X_{0}, X_{1}, \ldots, X_{g-1}, Y_{0}, Y_{1}, \ldots, Y_{g-1}$ such that $X_{i} \in M^{i}$ and $Y_{i} \in(V M)^{i}$ for all $i=0, \ldots, g-1$, and the Frobenius operator $F$ has the following normal form:

$$
\left(\begin{array}{ccccccccc}
0 & 0 & \ldots & 0 & 0 & 0 & \ldots & 0 & -p \\
1 & 0 & \ldots & 0 & p a_{2,2} & p a_{2,3} & \ldots & p a_{2, g} & 0 \\
\vdots & \ddots & \ddots & \vdots & \vdots & \ddots & \ddots & \vdots & \vdots \\
0 & \ldots & 1 & 0 & p a_{g, 2} & p a_{g, 3} & \ldots & p a_{g, g} & 0 \\
& & & & & & & & \\
0 & \ldots & 0 & 1 & 0 & 0 & \ldots & \ldots & 0 \\
0 & \ldots & \ldots & 0 & p & 0 & \ldots & \ldots & 0 \\
0 & \ddots & \ddots & 0 & 0 & p & \ldots & \ldots & 0 \\
\vdots & \ddots & \ddots & \vdots & \vdots & \vdots & \ddots & \vdots & \vdots \\
0 & \ldots & \ldots & 0 & 0 & \ldots & \ldots & p & 0
\end{array}\right)
$$

where $a_{i, j}=0$ if $i \not \equiv j(\bmod f)$ and $a_{i, j}=a_{j, i}$ for all $i, j$ between 2 and $g$.

Proof. We prove the following statement by induction

$\left(I_{n}\right)$ : There is an $X \in M^{0}$ and $Y_{i} \in(V M)^{i}$ for $i=0, \ldots, g-1$ such that

$$
Y_{0} \equiv F^{g} X \quad\left(\bmod p^{n} M\right), \quad Y_{g-1} \equiv-V X \quad\left(\bmod p^{n} M\right)
$$

and if we set $X_{i}:=F^{i} X$ for $i=0, \ldots, g-1$, then $\left\{X_{i}, Y_{i}\right\}$ is a symplectic basis modulo $p^{n} M$.

As $a(M)=1, M / p M$ is generated by one element $x$ over $k[F, V]$; it is generated over $k$ by $x, F^{1} x, \ldots, F^{g-1} x, V x, \ldots, V^{g-1} x$, and $F^{g} x$ (or $V^{g} x$ ). Particularly $F^{g}(M / p M$ ) is one-dimensional. Since the polarization is separable, it induces a perfect pairing

$$
M /(F, V) M \times F^{g}(M / p M) \rightarrow W / p W \simeq k .
$$

Thus there exists an element $X \in M^{0}$ such that $\left\langle X, F^{g} X\right\rangle \not \equiv 0 \bmod p$. As $k$ is algebraically closed, we may adjust $X$ by a scalar multiple such that $\left\langle X, F^{g} X\right\rangle \equiv 1 \bmod p$. This yields $\left\langle X,-V^{g} X\right\rangle \equiv 1$ $\bmod p$.

As $-V^{g} \bar{X} \in F^{g} \bar{M}$, where $\bar{M}:=M / p M$, we obtain $-V^{g} X \equiv F^{g} X \bmod p M$. Define

$$
Y_{0}:=F^{g} X, \quad Y_{i}:=-V^{g-i} X, \quad X_{i}:=F^{i} X \quad \forall i=1, \ldots, g-1 .
$$

Then we have $X_{i} \in M^{i}, Y_{i} \in(V M)^{i}$ and

$$
\left\langle X_{i}, Y_{j}\right\rangle \in \begin{cases}p W & \text { if } i>j \\ 1+p W & \text { if } i=j \\ W & \text { otherwise. }\end{cases}
$$

Set $X^{\prime}=X+a_{1} Y_{f}+\cdots+a_{r-1} Y_{(r-1) f}$. It follows from (2) that the $(r-1) \times(r-1)$ matrix $\left(\left\langle Y_{i f}, X_{j f}\right\rangle\right)_{1 \leq i, j<r}$ is non-degenerate $\bmod p$; we can find solutions $a_{i} \in W$ for $1 \leq i<r$ such that 
$\left\langle X^{\prime}, X_{j f}\right\rangle \equiv 0 \bmod p W$ for $j=1, \ldots, r-1$. Note that $F^{i} X^{\prime} \equiv F^{i} X \bmod p M$. Replacing $X$ by $X^{\prime}$, we have $\left\langle X, F^{i} X\right\rangle \equiv 0 \bmod p W$ for $0 \leq i<g$. Set $X_{0}:=X$ and re-define $Y_{i}$ and $X_{i}$ by (II). Thus we have verified $n=1$ of the statement $\left(I_{n}\right)$.

Suppose that we have a basis $\left\{X_{i}, Y_{i}\right\}$ satisfying $\left(I_{n}\right)$, we want to find a new basis $\left\{X_{i}^{\prime}, Y_{i}^{\prime}\right\}$ that satisfies $\left(I_{n+1}\right)$ and that $X_{i}^{\prime} \equiv X_{i}, Y_{i}^{\prime} \equiv Y_{i}\left(\bmod p^{n} M\right)$. Let

$$
X^{\prime}:=X+a_{1} p^{n} Y_{f}+a_{2} p^{n} Y_{2 f}+\cdots+a_{(r-1)} p^{n} Y_{(r-1) f}
$$

Since $F^{i} X^{\prime} \equiv F^{i} X \bmod p^{n+1} M$ for $i>0$, we have $\left\langle X^{\prime}, F^{i} X^{\prime}\right\rangle \equiv\left\langle X^{\prime}, X_{i}\right\rangle \bmod p^{n+1} W$. Dividing $\langle$,$\rangle by p^{n}$, we get a system of linear equations $\bmod p$ with coefficients $\left\langle Y_{j}, X_{i}\right\rangle$. Therefore we can find $a_{i}$, unique modulo $p$, such that $\left\langle X^{\prime}, F^{i} X^{\prime}\right\rangle \equiv 0 \bmod p^{n+1} W$ for $i=1, \ldots, g-1$. Clearly, $X^{\prime} \equiv X \bmod p^{n} M$.

Suppose $\left\langle X^{\prime}, F^{g} X^{\prime}\right\rangle \equiv 1+a p^{n} \bmod p^{n+1} W$. Choose $b \in W$ so that $b+b^{\sigma^{g}}+a \equiv 0 \bmod p$. Set $X^{\prime \prime}:=\left(1+b p^{n}\right) X^{\prime}$, then we have $\left\langle X^{\prime \prime}, F^{g} X^{\prime \prime}\right\rangle \equiv 1 \bmod p^{n+1} W$ from the choice of $b$. Replacing $X^{\prime}$ by $X^{\prime \prime}$, we find an $X^{\prime}$ satisfying

$$
\begin{aligned}
\left\langle X^{\prime}, F^{i} X^{\prime}\right\rangle & \equiv 0 \quad \bmod p^{n+1} W \quad \text { for } 0<i<g, \\
\left\langle X^{\prime}, F^{g} X^{\prime}\right\rangle & \equiv 1 \quad \bmod p^{n+1} W,
\end{aligned}
$$

and $X^{\prime} \equiv X \bmod p^{n} M$

Set $X_{i}^{\prime}:=F^{i} X^{\prime}, Y_{g-1}^{\prime}:=-V X^{\prime}, Y_{0}^{\prime}:=F^{g} X^{\prime}$. We can easily adjust the rest of $Y_{i}$ by adding some linear combinations of $p^{n} Y_{i}$, and get $Y_{i}^{\prime}$ for $i=1, \ldots, g-2$ such that $\left\{X_{i}^{\prime}, Y_{i}^{\prime}\right\}$ satisfies $\left(I_{n+1}\right)$.

By completeness of $M$, we get a symplectic basis $\left\{X_{i}, Y_{i}\right\}$ with

$$
(*): \quad X_{i} \in M^{i}, Y_{i} \in(V M)^{i}, X_{i}=F^{i} X_{0}, Y_{0}=F^{g} X_{0}, \text { and } Y_{g-1}=-V X .
$$

It follows from $(*)$ that $F Y_{j}=p Y_{j+1}+\sum_{i=1}^{g-1} p a_{i+1, j+2} X_{i}$ for $j=0, \ldots, g-2$ such that $a_{i+1, j+2}=$ 0 except $i+1 \not \equiv j+2 \bmod f$. That is, $a_{i, j} \neq 0$ only when $i \equiv j(\bmod f)$.

\section{$\S 5$. Local moduli of the Newton strata}

(5.1) We review the method of Cayley-Hamilton developed in Oort [16]. Let $M$ be a Dieudonné module of rank $2 g$. Suppose that the representative matrix of the Frobenius operator on $M$ with respect to a basis has the following form (cf. Theorem 4.5)

$$
\left(\begin{array}{ccc}
B & C & E \\
0 & D & 0
\end{array}\right)
$$

where $B$ is the $g \times(g-1)$ matrix with entries $b_{i+1, j}=\delta_{i j}, D$ is the $g \times g$ diagonal matrix $\operatorname{diag}(1, p, p, \ldots, p), E$ is the column vector $(-p, 0, \ldots, 0)^{t}$ and $C$ is a $g \times g$ matrix of the form

$$
\left(\begin{array}{cccc}
a_{1,1} & p a_{1,2} & \ldots & p a_{1, g} \\
a_{2,1} & p a_{2,2} & \ldots & p a_{2, g} \\
\vdots & \vdots & \ddots & \vdots \\
a_{g, 1} & p a_{g, 2} & \ldots & p a_{g, g}
\end{array}\right) .
$$


The matrix $C$ is the main part of the representative matrix. The assertion of this method says that the Newton polygon of $M$ can be read off from that of the following polynomial 16, Proposition $2.7]$

$$
X^{2 g}+\sum_{k=g-1}^{0} \sum_{i=1}^{g-k} p^{i-1} a_{i+k, i}^{\sigma^{g-i+1}} X^{g+k}+\sum_{k=1}^{g-1} \sum_{i=1}^{g-k} p^{i+k-1} a_{i, i+k}^{\sigma^{g-i-k+1}} X^{g-k}-p^{g} .
$$

Suppose that every $a_{i, j}$ is either a unit, or sufficiently close to 0 in $W$. In this case the valuation of the coefficient of every $X^{g+k}$ (resp. every $\left.X^{g-k}\right)$ is $\operatorname{ord}\left(p^{i-1} a_{i+k, k}\right)\left(\operatorname{resp} \operatorname{ord}\left(p^{i+k-1} a_{i, i+k}\right)\right)$ for the smallest $i$ such that $a_{i+k, i}$ (resp. $a_{i, i+k}$ ) is a unit. Then the Newton polygon of $M$ is the lower convex polygon obtained by removing from the following digram the $a_{i, j}$ with $p \mid a_{i, j}$

$$
\begin{aligned}
& -1 \\
& \begin{array}{llll}
a_{g, g} & \ldots & \ldots & a_{1, g}
\end{array} \\
& \begin{array}{llll}
1 & a_{g, 1} & \ldots & a_{1,1}
\end{array}
\end{aligned}
$$

where numbers are placed on the lattices of a $2 g \times g$ box, -1 (resp. 1) is placed at $(2 g, g)$ (resp. $(0,0))$.

(5.2) We recall the deformation theory of Dieudonné modules developed in Norman [13] and Norman-Oort [14. We follow the convenient setting of [13, Section 0] and [3, Section 2, p. 1011].

Let $R$ be a commutative ring of characteristic $p$. Let $W(R)$ denote the ring of Witt vectors over $R$, equipped with the Verschiebung $\tau$ and Frobenius $\sigma$ :

$$
\begin{aligned}
& \left(a_{0}, a_{1}, \ldots\right)^{\tau}=\left(0, a_{0}, a_{1}, \ldots\right) \\
& \left(a_{0}, a_{1}, \ldots\right)^{\sigma}=\left(a_{0}^{p}, a_{1}^{p}, \ldots\right) .
\end{aligned}
$$

Let $\operatorname{Cart}_{p}(R)$ denote the Cartier ring $W(R)[F] \llbracket V \rrbracket$ modulo the relations

- $F V=p$ and $V a F=a^{\tau}$,

- $F a=a^{\sigma} F$ and $V a^{\tau}=a V, \forall a \in W(R)$.

A left $\operatorname{Cart}_{p}(R)$-module is uniform if it is complete and separated in the $V$-adic topology. A uniform $\operatorname{Cart}_{p}(R)$-module $M$ is reduced if $V$ is injective on $M$ and $M / V M$ is a free $R$-module. A Dieudonné module over $R$ is a finitely generated reduced uniform $\operatorname{Cart}_{p}(R)$-module.

There is an equivalence of categories between the category of finite dimensional commutative formal groups over $R$ and the category of Dieudonné modules over $R$. We denote this functor by $\mathbf{D}_{*}$. The tangent space of a formal group $G$ is canonically isomorphic to $\mathbf{D}_{*}(G) / V \mathbf{D}_{*}(G)$.

(5.3) Let $\underline{M}_{0}=\left(M_{0},\langle\rangle,, \iota\right)$ be a local-local separably quasi-polarized Dieudonné $\mathcal{O}$-module with $a\left(M_{0}\right)=1$. For simplicity we assume that $\underline{a}\left(\underline{M}_{0}\right)=(1,0, \ldots, 0)$. Let $\left\{X_{i}, Y_{i}\right\}$ be a symplectic basis as in Theorem 4.5, Set

$$
\begin{array}{ll}
e_{\mathrm{k}}^{\ell}:=X_{(\mathrm{k}-1) f+\ell} & \text { for } \quad 0 \leq \ell \leq f-1,1 \leq \mathrm{k} \leq r, \\
f_{\mathrm{k}}^{\ell}:=Y_{(\mathrm{k}-1) f+\ell} & \text { for }-1 \leq \ell \leq f-2,1 \leq \mathrm{k} \leq r,
\end{array}
$$


with the notation $X_{i \pm g}=X_{i}$ and $Y_{i \pm g}=Y_{i}$. Then $\left\{e_{\mathrm{k}}^{i}, f_{\mathrm{k}}^{i}\right\}_{1 \leq \mathrm{k} \leq r}$ is a symplectic $W$-basis of $M^{i}$ for each $i \in I$. It is convenient to extend the notation $f_{\mathrm{k}}^{\ell}$ and $e_{\mathrm{k}}^{\ell}$ for all integers $\ell, \mathrm{k}$ by the rule (5). We have

$$
\begin{aligned}
& F e_{j}^{\ell}=e_{j}^{\ell+1} \quad \text { except }(\ell, j)=(f-1, r), \\
& F e_{r}^{f-1}=f_{1}^{0}, \\
& F f_{j}^{\ell}=p f_{j}^{\ell+1}+\sum_{i=1}^{r} p a_{i, j}^{\ell+1} e_{i}^{\ell+1} \quad \text { except }(\ell, j)=(-1,1), \\
& F f_{1}^{-1}=-p e_{1}^{0} .
\end{aligned}
$$

(5.4) Let $\operatorname{Def}\left[\underline{M}_{0}\right]$ be the deformation functor of $\underline{M}_{0}$. This functor is smooth; moreover, for any local Artinian $k$-algebra $(R, \mathfrak{m})$ with residue field $k$ there is a bijection [13]

$$
\operatorname{Def}\left[\underline{M}_{0}\right](R) \simeq \operatorname{Hom}_{\mathcal{O}}\left(V M_{0} / p M_{0}, M_{0} / V M_{0}\right)^{\mathrm{sym}} \otimes_{k} \mathfrak{m} .
$$

Here we identify $V M_{0} / p M_{0}$ with the dual $\left(M_{0} / V M_{0}\right)^{*}$ by the pairing $\langle$,$\rangle . Let \left\{\left(e_{\mathrm{k}}^{\ell}\right)^{*},\left(f_{\mathrm{k}}^{\ell}\right)^{*}\right\}$ be the dual basis for the basis $\left\{e_{\mathrm{k}}^{\ell}, f_{\mathrm{k}}^{\ell}\right\}$. We may identify $\left(e_{\mathrm{k}}^{\ell}\right)^{*}$ with $f_{\mathrm{k}}^{\ell}$ and $\left(f_{\mathrm{k}}^{\ell}\right)^{*}$ with $e_{\mathrm{k}}^{\ell}$. Thus any homomorphism that sends $\bar{f}_{j}^{\ell} \mapsto \sum t_{i, j}^{\ell} \bar{e}_{i}^{\ell}$ with $t_{i, j}^{\ell}=t_{j, i}^{\ell} \in \mathfrak{m}$ gives rise to a deformation of $\underline{M}_{0}$ over $R$; and conversely any deformation of $\underline{M}_{0}$ over $R$ arises from such a unique homomorphism. Hence

$$
R_{\text {univ }}:=k\left[\left|t_{i, j}^{\ell}\right|\right] /\left(t_{i, j}^{\ell}-t_{j, i}^{\ell}\right)=k\left[\left|t_{i, j}^{\ell}\right|\right]_{0 \leq \ell<f, 1 \leq j \leq i \leq r}
$$

is the universal deformation ring of $\underline{M}_{0}$.

By [13] or [14, the universal deformation $M_{R_{\text {univ }}}$ of $\underline{M}_{0}$ over $R_{\text {univ }}$ is given by the following generators and relations:

$$
\begin{aligned}
F e_{j}^{\ell} & =e_{j}^{\ell+1} \quad \text { except }(\ell, j)=(f-1, r), \\
F e_{r}^{f-1} & =f_{1}^{0}+\sum_{i=1}^{r} T_{i, 1}^{0} e_{i}^{0}, \\
f_{j}^{\ell} & =V\left(f_{j}^{\ell+1}+\sum_{i=1}^{r}\left(a_{i, j}^{\ell+1}+T_{i, j}^{\ell+1}\right) e_{i}^{\ell+1}\right) \quad \text { except }(\ell, j)=(-1,1), \\
f_{1}^{-1} & =-V e_{1}^{0},
\end{aligned}
$$

where $T_{i, j}^{\ell}:=\left(t_{i, j}^{\ell}, 0, \ldots\right)$ is the Teichmüller lifting of $t_{i, j}^{\ell}$ for each $\ell, i, j$.

(5.5) Let $\underline{G}_{0}$ be the $p$-divisible group with additional structures corresponding to $\underline{M}_{0}$ and $\underline{G}^{\text {univ }}$ be its universal deformation over $\mathcal{U}:=\operatorname{Spec} R_{\text {univ }}$. Let $\beta_{0}=N P\left(M_{0}\right)$ and $\beta$ be an admissible Newton polygon below $\beta_{0}$. Denote by $\mathcal{U} \geq \beta$ the reduced closed subscheme of $\mathcal{U}$ whose points has Newton polygon lying over or equal to $\beta$. By the theory of Cayley-Hamilton, the closed Newton stratum $\mathcal{U}^{\geq \beta}$ is defined by the equations $t_{i, j}^{\ell}$ whose corresponding "position" in the Oort diagram (44) of (5.1)) is strictly below the Newton polygon $\beta$; see the discussion in Oort [16 Section 3]; also see an example in (5.7). 
From (3) of (5.1) and (6) of (5.4), it is not hard to see that the corresponding position of $t_{\mathrm{k}+j, j}^{\ell}$ is $((r-\mathrm{k}) f,(j-1) f+\ell)$. Put

$$
S(\beta):=\left\{t_{i, j}^{\ell} ; j \leq i \text { and the position of } t_{i, j}^{\ell} \text { is higher or on } \beta\right\},
$$

thus $\mathcal{U}^{\geq \beta} \simeq \operatorname{Spec} k[|t|]_{t \in S(\beta)}$.

When $\beta_{0}$ is supersingular, $\mathcal{U} \geq \beta_{0}$ is the supersingular locus in $\mathcal{U}$. One computes that

$$
\operatorname{dim} \mathcal{U}^{s s}=\left|S\left(\beta_{0}\right)\right|=\sum_{i=1}^{r}[i f / 2]= \begin{cases}\mathrm{k} \frac{r(r+1)}{2} & \text { if } f=2 \mathrm{k}, \\ \mathrm{k} \frac{r(r+1)}{2}+\left[r^{2} / 4\right] & \text { if } f=2 \mathrm{k}+1 .\end{cases}
$$

(5.6) Theorem Let $\underline{M}_{0}$ be a separably quasi-polarized Dieudonné $\mathcal{O}$-module with $a\left(M_{0}\right)=1$ and $\beta \leq N P\left(M_{0}\right)$ be an admissible Newton polygon. Then the closed Newton stratum $\mathcal{U} \geq \beta$ of the universal deformation $\mathcal{U}$ is formally smooth of dimension $|S(\beta)|$ above; the generic point has Newton polygon equal to $\beta$. If $M_{0}$ is supersingular, the supersingular locus $\mathcal{U}^{\text {ss }}$ in $\mathcal{U}$ has dimension

$$
\operatorname{dim} \mathcal{U}^{s s}= \begin{cases}\mathrm{k} \frac{r(r+1)}{2} & \text { if } f=2 \mathrm{k} \\ \mathrm{k} \frac{r(r+1)}{2}+\left[r^{2} / 4\right] & \text { if } f=2 \mathrm{k}+1 .\end{cases}
$$

We have done the case where $M_{0}$ is local-local. When $M_{0}$ is not local-local, we have a decomposition $M_{0}=M_{0}^{\text {loc-loc }} \oplus M_{0}^{\text {ord }}$. Write $\mathcal{U}_{1}$ for the universal deformation for $M_{0}^{\text {loc-loc }}$ and $\beta=N P\left(M_{0}^{\text {ord }}\right) \cup \beta_{1}$. The defining equations for $\mathcal{U} \geq \beta$ are the same as those for $\mathcal{U}_{1}^{\geq \beta_{1}}$. Therefore, we obtain the same dimension formula as before.

(5.7) Example. When $f=3, r=2$, the main part of the representative matrix of the Frobenius operator on the universal Dieudonné module is

$$
\left(\begin{array}{llllll}
T_{1,1}^{0} & & & & & \\
& & & & \\
& p\left(a_{1,1}^{1}+T_{1,1}^{1}\right) & & & \\
& & p\left(a_{1,1}^{2}+T_{1,1}^{2}\right) & & & \\
T_{2,1}^{0} & & & p\left(a_{1,2}^{1}+T_{1,2}^{1}\right) & \\
& p\left(a_{2,1}^{1}+T_{2,1}^{1}\right) & & & & \\
& & p\left(a_{2,1}^{2}+T_{2,1}^{2}\right) & & p\left(a_{2,2}^{1}+T_{1,2}^{2}\right) \\
& & & & p\left(a_{2,2}^{2}+T_{2,2}^{2}\right)
\end{array}\right)
$$

We have the following picture

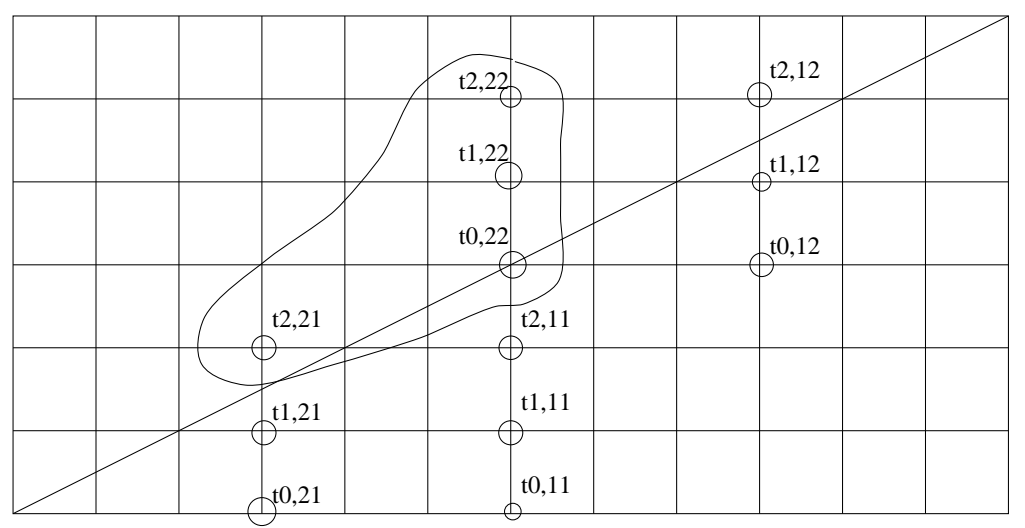


Here $t 2,11$ denotes $t_{1,1}^{2}$ etc. Particularly $\mathcal{U}^{s s} \simeq \operatorname{Spec} k\left[\left|t_{2,1}^{2}, t_{2,2}^{0}, t_{2,2}^{1}, t_{2,2}^{2}\right|\right]$ when $M_{0}$ is supersingular.

(5.8) We now return to complete Theorem 1.2 1.4 Using the Serre-Tate theorem, the deformation problem is reduced to that for $p$-divisible groups with the additional structure. As $p \nmid \Delta$, one reduces to that for quasi-polarized $p$-divisible $\mathcal{O}$-groups by the Morita equivalence. By Theorem 5.6 Theorem 1.2 is proved.

Using Theorem 1.1 one has a separably polarized supersingular point $\underline{A}_{0}$ in the open subset $U$. Then Theorem 1.3 follows from Theorem 1.2 immediately by induction.

Theorem 1.4 follows from Theorem 1.3 and Theorem 1.1](2).

\section{References}

[1] O. Bültel and T. Wedhorn, Congruence relations for Shimura varieties associated to some unitary groups. To appear Journal de l'Institut de Math. de Jussieu.

[2] C.-L. Chai, Newton polygons as lattice points. Amer. J. Math. 122 (2000), 967-990.

[3] C.-L. Chai and P. Norman, Singularities of the $\Gamma_{0}(p)$-level structure. J. Algebraic Geom. 1 (1992), 251-178.

[4] A.J. de Jong and F. Oort, Purity of the stratification by Newton polygons. J. Amer. Math. Soc. 13 (2000), 209-241.

[5] P. Deligne and G. Pappas, Singularités des espaces de modules de Hilbert, en les caractéristiques divisant le discriminant. Compositio Math. 90 (1994), 59-79.

[6] E.Z. Goren and F. Oort, Stratifications of Hilbert modular varieties in positive characteristic. J. Algebraic Geom. 9 (2000), 111-154.

[7] A. Grothendieck, Groupes de Barsotti-Tate et Cristaux de Dieudonné. Les Presses de l'Université de Montréal, 1974.

[8] N. M. Katz, Slope filtration of F-crystals. Journées de Géométrie. Algébrique de Rennes (1978), Vol I, pp. 113-163, Astérisque, no. 63, Soc. Math. France, Paris, 1979.

[9] R. E. Kottwitz, Isocrystals with additional structure. Compositio Math. 56 (1985), 201-220.

[10] R. E. Kottwitz, Points on some Shimura varieties over finite fields. J. Amer. Math. Soc. 5 (1992), 373-444.

[11] W. Messing, The crystals associated to Barsotti-Tate groups: with applications to abelian schemes. Lecture Notes in Math. 264, Springer-Verlag, 1972.

[12] B. Moonen, A dimension formula for Ekedahl-Oort strata. To appear Ann. Inst. Fourier (Grenoble). 
[13] P. Norman, An algorithm for computing moduli of abelian varieties. Ann. Math. 101 (1975), 499-509.

[14] P. Norman and F. Oort, Moduli of abelian varieties, Ann. Math. 112 (1980), 413-439.

[15] F. Oort, Moduli of abelian varieties and Newton polygons. C. R. Acad. Sci. Paris Sér. I Math. 312 (1991), 385-289.

[16] F. Oort, Newton polygons and formal groups: conjectures by Manin and Grothendieck. Ann. Math. 152 (2000), 183-206.

[17] F. Oort, Newton Polygon strata in the moduli space of abelian varieties. Moduli of Abelian Varieties, 417-440. (ed. by C. Faber, G. van der Geer and F. Oort), Progress in Mathematics 195, Birkhäuser 2001.

[18] M. Rapoport, Compactifications de l'espaces de modules de Hilbert-Blumenthal. Compositio Math. 36 (1978), 255-335.

[19] M. Rapoport, On the local zeta function of quaternionic Shimura varieties with bad reduction. Math. Ann. 279 (1988), 673-697.

[20] M. Rapoport, On the Newton stratification. Sém. Bourbaki Exp. 903 (2001-2002), 18 pp, March, 2002.

[21] M. Rapoport, A guide to the reduction modulo $p$ of Shimura varieties, Preprint, math.AG/0205022,

[22] M. Rapoport and M. Richartz, On the classification and specialization of $F$-isocrystals with additional structure. Compositio Math. 103 (1996), 153-181.

[23] M. Rapoport and Th. Zink, Period Spaces for p-divisible groups. Ann. Math. Studies 141, Princeton Univ. Press, 1996.

[24] T. Wedhorn, Ordinariness in good reductions of Shimura varieties of PEL-type. Ann. Sci. École Norm. Sup. (4) 32 (1999), 575-618.

[25] C.-F. Yu, On the supersingular locus in Hilbert-Blumenthal 4-folds. J. Algebraic Geom. 12 (2003), 653-698.

[26] C.-F. Yu, On reduction of Hilbert-Blumenthal varieties. Ann. Inst. Fourier (Grenoble) 53 (2003), 2105-2154.

[27] T. Zink, Cartiertheorie kommutativer formaler Gruppen. Teubner-Texte Math. Teubner, Leipzip, 1984.

Institute of Mathematics

Academia Sinica

128 Academia Rd. Sec. 2, Nankang

Taipei, Taiwan 115

E-mail Address: chiafu@math.sinica.edu.tw 